Proceedings

\title{
Concordance between self-reported and national health insur- ance administrative data for care continuity measurements
}

\author{
I-Jen Wang *, Ren-Hui Zhong, Christy Pu and Kuan-Chia Lin \\ * Correspondence: wij636@gmail.com
}

\begin{abstract}
Continuity of care (COC) is regarded as an important factor to improve the quality of healthcare. In this study, we want to assess the concordance between the continuity of child care measured by administrative data and the questionnaire reported by caregivers. In addition, we also analyze the related factors that affect the continuity of child care reported by caregivers in the questionnaire. The population profile of the 2016 National Health Insurance Research Database (NHID) and the child care questionnaire was linked to analyze 1283 caregivers of children, using the four indexes UPC (Usual Provider of Care), HI (Herfindahl Index), COCI (Continuity of Care Index), and SECON (Sequential Continuity Index) to calculate the continuity of care. Ordinal logistic regression used to examine the association between the two measurements. Then, 1454 caregivers of children, and the regression models were developed by using the demographic characteristics of caregivers and children to explore the factors that affect the continuity of child care reported by the caregivers. The results of the study indicated that the highest degree of fit is the COCI index. In the five aspects of the questionnaire, the caregiver reported children's loyalty to the doctor for the same disease (OR=2.54, 95\%CI: 1.40-4.62), loyalty to the doctor for different diseases (OR=1.93, 95\%CI: 1.06-3.52), trust (OR=3.16, 95 \%CI: 1.76-5.69) and importance (OR=2.91; 95\%CI: 1.21-7.36) four aspects are statistically significant. The satisfaction of the health-care system is the most important factor affecting the continuity of child care. When caregivers are highly satisfied, they have a positive impact on the confidence of the visiting physician, the importance of having a regular care physician for children, the frequency of changing physicians, and the willingness to pay for healthcare.
\end{abstract}

Publisher's Note: MDPI stays neutral with regard to jurisdictional claims in published maps and institutional affiliations.

\section{(c) (i)}

Copyright: $@ 2020$ by the authors. Submitted for possible open access publication under the terms and conditions of the Creative Commons Attribution (CC BY) license (http://creativecommons.org/licenses/by/4.0/).
Keywords: Continuity of care; caregivers' perspective; concordance 